\title{
ADVANCES IN COMPUTER CHESS CONFERENCE 7
}

\author{
Christian Posthoff and Michael Schlosser \\ Technische Universität Chemnitz \\ Germany
}

The Advances in Computer Chess Conference is a triennial event. The $7^{\text {th }}$ instance took place at the University of Limburg in Maastricht under the generous sponsorship of Interpolis, the Municipality of Maastricht, the Rabobank and the Max Euwe-centrum. Of course, the ICCA provided the tutelary aegis. On the $1^{\text {st }}$ and $2^{\text {nd }}$ of July 1993, the conference was chaired by Professor H.J. van den Herik, who is also the national President of the Dutch Association for Artificial Intelligence. In the issues dealt with and the approaches adopted, this confluence of computer chess and artificial intelligence was most gratifying, as was the attendence of over 60 delegates.

\section{Botvinnik as the Guest of Honour}

The conference had succeeded in inviting, as its Guest of Honour, Professor Mikhail Botvinnik, still hale and hearty after 82 years, whose personality shone brilliantly even though his contribution failed to coincide with the special interests of most delegates. Still, the Guest of Honour was universally admired for being, in a very proper sense, chess history incarnate, remembering all World Champions personally barring Steinitz. To him major tournaments going back to 1936 (Moscow and Nottingham) are still vivid when he reminisces; of course, he has also earned his spurs in the more limited field of computer chess by papers going back to 1960 .

Indeed, Shannon's seminal paper of 1950 was recalled in Dr. Botvinnik's keynote lecture entitled Shannon's Problem and How to Solve it. He summarized his plans and surveyed the results achieved so far by his team. Whatever the final outcome, nobody could fail to be impressed by his iron will to find a way toward reaching such ambitious goals. To paraphrase him: "It is not important whether a computer program will win a match with the World Champion - that is only the Champion's problem. It is far more important to transfer the methods used to the problems in law, economics, society at large, and politics, which are of ever-increasing complexity. Solving them is crucial to the well being of humanity as a whole. Here, the help of intelligent computer systems is indispensable - such systems must adopt human and rational principles, emulate human beings and support them in all the relevant areas. This is the real goal; plans for creating an artificial grandmaster operating in similarity to human thinking is an interesting subgoal, a fascinating area for experimentation as well as a major component, as a model, in AI activities."

This role of AI served to unify the whole conference; even when matters of chess dominated the themes of many lectures, the conference as a whole could be viewed, with justice, as dealing with applied artificial intelligence to which the central question is determining what means and methods should be used for solving any given problem. Our survey of the contributions will adopt this perspective.

\section{The first day: presentations and representations}

F. Gobet and P. Jansen (Carnegie-Mellon University): Towards a Chess Program Based on a Model of Human Memory. This presentation introduced CHUMP, a chess program learning piece patterns, proceeding to associate moves and move sequences (i.e., tactical and strategic ideas with these patterns) and deducing potential good moves from these associations. Recorded grandmaster games served to identify the patterns as well as to test the results. The experiments show a tendency for program performance to be improved by this method, which serves as an indication that eventually it has the potential for yielding a competitive chess program. Generalizing this result, we find that pattern-driven strategies are derivable and 
implementable, though not without many obstacles. This applies to chess as well as to other domains and the authors recommend careful analysis of the chess application before the method is transferred to other domains too enthusiastically and without further checks.

A research group of the University of Paderborn (R. Feldmann, P. Mysliwietz and B. Monien), having taken the second place in the computer-chess world championship in Madrid in November 1992, exhibited results in parallelizing algorithms (Game-Tree Search on a Massively Parallel System). The program being run on transputer systems of 256 to 1024 processors, the shape of the networks, in dependence of the tasks to be performed, turned out to be a subtle and decisive subject for investigation (see also ICCA Journal, Vol. 12, No. 2, pp. 65-73).

The invited lecture was by IGM dr. J. Nunn (Extracting Information from an Endgame Database) and dealt with transfering an endgame database, containing many millions of bytes and, by definition, the complete knowledge for a specific endgame, into a form useful to human players. Nunn exhibited his partial solution for one such endgame proceeding from Thompson's database for the KRPKR endgame. All possible positions are broken down by type, and strategies for each of them are exhibited. In contrast to all other books, the analyses given are not more or less conjectural but guaranteed as indisputable truths (see also ICCA Journal Vol. 15, No. 2, pp. 75-76 and Vol. 16, No. 2, pp. 94-95).

The reviewers of this conference feel that the contributions by Gobet and Jansen on the one hand and by Nunn on the other are complimentary in a most interesting way. Whereas the former starts from examples ans attempts generalization to rules, the latter tackles the converse problem: how to condense a gigantic amount of miniscule knowledge into rules and patterns. Specifically, Nunn's contribution is transferable as a problem statement: Given complete knowledge in a domain, obtained where necessary by variegated means, how does one transform such knowledge into a representation utilizable by human beings while preserving its full validity.

Interesting questions of using time-restricted resources came to the fore in On Timing and Permanent Brain in Computer Chess (I. Althöfer, Bielefeld; Chr. Donninger, Vienna; U. Lorenz and V. Rottmann, Paderborn). They investigate normal timing (40 moves/60 minutes), constant timing (90 s/move) as well as the utilization of the opponent's time and how these factors affect playing strength. The idea of a "3-brain", two chess computers under a single human supervisor, is explored empirically; as may happen to any good piece of research, the investigation raises more new questions than it solves.

Acquisition, Transformation, and Utilization of Knowledge Representations (Chr. Posthoff, M. Schlosser, R. Staudte and J. Zeidler, TU Chemnitz) demonstrated how different knowledge representations can be derived from endgame databases and mutually transformed, adopting them to specific requirements. There is a connection here with the acquisition, evaluation and tuning of evaluation functions, a long-term research theme of the presentators.

A press conference, a spectacular game of J. Nunn against the computer-chess world champion Chessmachine WK 1992 (operated by J. Louwman) and a reception (with opinions and gossip flashing back and forth) concluded the first day.

The game Nunn vs. Chessmachine WK, with 30 minutes for either player, follows.

Nunn - Chessmachine WK 1992 (NicKey KP 3.6)

1. e4 e5 2. Nf3 Nf6 3. Nc3 Nc6 4. Bb5 Bb4 5. d3 d6 6. 0-0 0-0 7. Bg5 Bxc3 8. bxc3 h6 9. Bh4 Qe7 10. Re1 Nd8 11. d4 Ne6 12. dxe5 dxe5 13. Nxe5 Qc5 14. Bxf6 Qxb5 15. a4 Qa5 16. Ng4 gxf6 17. e5 Qxc3 18. Nxh6+ Kh7 19. Ng4 fxe5 20. Nf6+ Kg7 21. Ne4 Qc6 22. Qg4+ Kh8 23. Qh4+ Kg8 24. Nf6+ Kg7 25. Nh5+ Kg6 26. Qf6+ Kh7 27. Rxe5 Rg8 28. Qxf7+ Kh8 29. Qf6+ Ng7 30. Qh4 Nf5 31. Qf6+ Qxf6 32. Nxf6 Rg6 33. Re8+ Kg7 34. Nd5 Nh4 35. g3 Nf3+ 36. Kh1 c6 37. Nc7 Rh6 38. h4 Rb8 39. Kg2 Nd4 40. Rd1 Ne6 41. Nxe6+ Rxe6 42. Red8 Re4 43. f3 Rxa4 44. g4 Rf4 45. h5 Rc4 46. g5 Rxc2+ 47. Kg3 Rc5 48. f4 Rc3+ 49. Kf2 Rc4 50. Kg3 a5 51. f5 b5 52. h6+ Kh7 53. Rh1 Rc3+ 54. Kf4 Rc4+ 55. Ke3 Rc3+ 56. Kf4 Rc4+ 57. Ke3 Rc3+ 1/2 


\section{The second day}

Endgame databases, tentatively moving towards practical relevance in computer chess, play already a major role in checkers, boding well for further developments (not only in chess and checkers). Solving Large Retrograde Analysis Problems Using a Network of Workstations (R. Lake, J. Schaeffer and P. Lu, University of Alberta) surveyed the various problems that present themselves in a realistic undertaking:

- in-game access to large databases (one 8-piece database comprises over 100 Gigabytes) - where can such data sets be stored while preserving timely accessibility?

- more sophisticated mechanisms for addressing in storage and retrieval;

- transferring to parallel systems (over 90 processors in a practical instance) and transferring to heterogeneous networks;

- granted that 9-piece databases are as yet infeasible, what will a full set of 8-piece databases contribute to program performance?

Obviously, the prime challenge here is to computer scientists, though many of the problems are of general relevance to the practicability of $\mathrm{AI}$ methods.

How Hard is the Correct Coding of an Easy Endgame (J.-C. Weill, Université Paris 8, Vincennes) showed an application of Quinlan's ID3 algorithm to a specific classification problem, viz. how to separate optimally won positions from those drawn.

Search procedures being currently so central in AI, a presentation of newly proposed developments in this domain was most fitting. Null Move and Singular Extension Search (T. Marsland and Chun Ye, University of Alberta) addressed new ideas in searching: the combination of a $d$-ply search and a $q$-ply null-move quiescence search, as well as Principal-Variation singular extensions and Fail-High singular extensions. The object of demonstration was Chinese chess.

A. Opdahl and B. Tessem (University of Bergen, Norway) in Long-Term Planning in Computer Chess exhibited a framework for using planning methods in chess programs. Ideally, appropriate plans have been stored for each stage of the game; these can be recognized, evaluated, modified and applied. Case-based reasoning, similarity of patterns, modification of strategies, and a plan-description language were topical in their lecture.

Random Evaluations in Chess by M. Smith and Don Beal (Queen Mary and Westfield College, London) surprised many delegates by its counter-intuitive approach. In the absence of more definite knowledge, random but legal successor nodes are probed and given a value according to a random (!) evaluation function on the sole condition that in this function the number of successor nodes is kept track of. Assigning positive weight to this number is roughly equivalent to positively appraising mobility; hence, the random procedure is not so random after all indeed may serve as a test vehicle for evaluation functions which, to be judged adequate, should at least outperform the random one.

A second lecture by T. Marsland (Parallelizing Alpha-Beta: a Conceptual Model) treated fundamental questions of parallel search methods. The triple goal of search

- without excessive communication,

- without excessive duplication,

- without excessive waiting

imposes a need to distribute the workload dynamically; the lecture convincingly showed that strategic games are essential for arriving at a clear formulation of the problem and are a useful vehicle for the evaluation of solutions found.

W. Pijls and A. de Bruin (Erasmus University, Rotterdam), also in the domain of search procedures, proposed a generalization of a well-known search method (Generalizing Alpha-Beta; see also ICCA Journal, Vol. 16, No. 1, pp. 18-30). 
It seems there is no end to improving search algorithms. A. Reinefeld (Paderborn) presented in A Minimax Algorithm Faster than Alpha-Beta two search algorithms, based on SSS* and Dual*, both being more effective than alpha-beta by node count. Delegates may be eager to test these methods for practical relevance.

Deviating from the central theme, though by a small margin only, were Ch. Posthoff, R. Staudte and M. Schlosser (Chemnitz) in their paper Chess Programming and Computer Science Education. They shed light on the pedagogical theme of game programming as an appetizing introduction to methods of AI, incidentally delivering interesting topics for programs and projects.

The fact that the host of the conference and his research team have contributed no fewer than three papers redounds greatly to his credit. H. Iida, J. Uiterwijk and H.J. van den Herik (Thoughts on the Application of Opponent-Model Search) insist that any model comprehensive enough to include evaluation should also include a model of the opponent's evaluation. Studying the latter will lead to two contrasting search trees the difference between which should be useful for identifying an opponent's weaknesses and, with this knowledge to guide one, to assess and, hence, balance risks against gains.

Orientation towards the human being also characterized Speculative Play in Computer Chess by J. Uiterwijk and H.J. van den Herik. Equally valued moves may acquire unequal characteristics by the opponent's fallible nature, e.g., the richness of the error spectrum, the probability of mating, his time-control problems if any, and many more. It is propososed to introduce bonus and malus functions to accommodate these practical differences within theoretical equality.

Proof-number search, invented in the Connect-Four and Go-Moku context, was shown to be transferable to chess. It was applied to chess-problem solving and exploited by L.V. Allis, D. Breuker and H.J. van den Herik in a presentation under the tempting title Mate in 38: Applying Proof-Number Search to Chess.

Even the best of things must come to an end; so did this conference, which has been of great benefit to the AI Community. Feeling we hold a brief for this community, we wish to express our admiration for the smoothest of all possible conference managements as well as for the kindness and hospitality of the Chairman, Professor Van den Herik and his team. Whether student or professor, café player or world champion, all delegates were made to feel they were honoured guests.

\section{PROCEEDINGS OF THE ACC7 CONFERENCE}

On July 1-2, 1993 the $7^{\text {th }}$ Advances in Computer Chess Conference was held in Maastricht, The Netherlands. The keynote lecture was by former Chess World Champion Prof.dr. M.M. Botvinnik and the invited lecture by IGM dr. John Nunn. Moreover, sixteen selected lectures on various aspects of computer chess were presented by leading researchers in the field.

All papers will be published in book form in early 1994 under the editorship of H.J. van den Herik, I.S. Herschberg and J.W.H.M. Uiterwijk. Its price through regular channels will be Dfl. 125.- (approximately $\$ 75$.- in the US).

To members of the ICCA the book is offered at the price of Dfl. 110.- (or US \$ 65.-), inclusive of postal delivery costs, if ordered before January 1, 1994. It is to our common advantage to combine your order with the renewal of your membership, since this will reduce banking fees. The usual conditions apply: your transfer should either be net to the ICCA or the amount should be increased to compensate for banker's fees to be incurred by us.

A simple mention "Proceedings ACC7" will effect your order. The book will be sent as soon as published to the address at which you receive the Journal. Should an other mailing address be used instead, please clearly state so. 